\title{
On invariance and Ricci-flatness of Hermitian metrics on open manifolds
}

\author{
Bert Koehler and Marco Kühnel*
}

\begin{abstract}
We discuss a technique to construct Ricci-flat hermitian metrics on complements of (some) anticanonical divisors of almost homogeneous complex manifolds and inquire into when this metric is complete and Kähler. This construction has a strong interplay with invariance groups of the same dimension as the manifold acting with an open orbit. Lie groups of this type we call divisorial. As an application we describe compact manifolds admitting a divisorially invariant Kähler metric on an open subset. Finally, we see a connection between the reducibility of the anticanonical divisor and the non-triviality of the Kähler cone on the complement.
\end{abstract}

Mathematics Subject Classification (2000). 32M05; 32M12; $14 \mathrm{M} 17$.

Keywords. Ricci-flat hermitian metrics, Kähler cone, almost homogeneous manifolds.

\section{Introduction}

The first problem addressed in this paper is the construction of Ricci-flat metrics on open complex manifolds. As a model for this situation serves the complement of a divisor on a compact manifold $X$. If $D \in\left|-K_{X}\right|$, then the section of $-K_{X}$ vanishing exactly on $D$ yields an isomorphism $\Omega_{X \backslash D}^{n} \cong \mathcal{O}_{X \backslash D}$. In analogy to the Calabi conjecture on compact manifolds this raises the expectation that there exists a complete Ricci-flat Kähler metric on $X \backslash D$. Moreover, methods to find such a metric may also work, if $D$ is not reduced, leading to the speculative existence of Ricci-flat Kähler metrics on $X \backslash D$, whenever $-\left(D+K_{X}\right)$ is effective. Part of this program has already been established. Tian and Yau have proved in [TY90] and [TY91] the existence of a complete Ricci-flat Kähler metric in case $D \in\left|-K_{X}\right|$ is neat, almost ample and smooth. Bando and Kobayashi have shown the claim, if $r D \in\left|-K_{X}\right|$ for $r>1$ and $D$ is ample, smooth and admits on itself a Kähler-Einstein metric. The metrics involved in the construction contain logarithmic terms. Of course, the techniques introduced in [TY90], [TY91] and [BK90] cannot be easily generalized to

\footnotetext{
*The authors acknowledge gratefully support by the DFG priority program 'Global Methods in Complex Geometry'.
} 
the reducible or non-reduced case. The method described in Section 1 does not care about this and is compatible with an algebraic structure. In particular, the metrics involved can be described in terms of polynomials, if $X$ is algebraic. Of course, this nice structure has a high prize: either $X \backslash D$ has trivial geometry and $D$ is expected to be 'very' reducible, or we have to drop the Kähler condition. Nevertheless, this approach shows some fundamental differences between the reducible and the smooth case of the divisor $D$.

The other problem is determining highly symmetric metrics on $X \backslash D$. Both problems get related by the idea that Ricci-flatness should be forced by a high order of symmetry. For example, a naive calculation shows that all metrics on $\mathbb{P}^{2} \backslash\{3$ general lines $\}$ invariant under the connected invariance group of the three lines are complete, Ricciflat and Kähler. In Chapter 2 we explain the connection between the construction in Chapter 1 and the symmetries of $D$ resp. symmetries of the metric. Here we discuss continuous symmetries. A striking point is that the metric is Kähler if and only if it is symmetric. Moreover, in this case the symmetry group is abelian. This allows a description of the manifolds admitting a divisorially invariant Kähler metric on an open subset; in particular, we recognize $D$ to be reducible or non-reduced, if $X$ is homogeneous and projective. By divisorial invariance we mean that the action of $G$ has an open orbit and $\operatorname{dim} G=\operatorname{dim} X$, if $G$ denotes the symmetry group of $D$. Three general lines in $\mathbb{P}^{2}$ satisfy this condition. Parts of the description are well known.

Winkelmann treated in [Wi04] the problem when $T_{X}(-\log D)$ is trivial. Of course, the condition that there is a Ricci-flat metric on $X \backslash D$ is much weaker than the triviality of $T_{X}(-\log D)$. However, in the respective Kähler cases there are great similarities. We will note this at the appropriate place.

As a last topic we inquire into a sort of Kähler classes of the constructed metrics. Two metrics shall be regarded as equivalent if they differ only by a Kähler potential. We call the cone of $G$-invariant metrics generated by this equivalence $K_{G}(X, D)$. If we denote $n:=\operatorname{dim} X$, then we will prove that $\operatorname{dim} K_{G}(X, D) \geq \frac{1}{2} n(n-1)$ for reduced $D$. So even if $\operatorname{dim} \operatorname{Alb}(X)=0$ the cone $K_{G}(X, D)$ is highly non-trivial. This effect for $K(X, D):=K_{1}(X, D)$ is in close relation with the reducibility of $D$. In the appendix we will show that $K(X, D)=0$, if $D$ is smooth and ample and $X$ has simple enough topology,e.g. $X=\mathbb{P}^{3}$.

The authors would like to thank the referee for pointing out a mistake in the appendix.

\section{Vocabulary}

We consider compact complex manifolds $X$. Of great importance will be the automorphism group $\operatorname{Aut}(X)$ and its action on $X$. If $G \subset \operatorname{Aut}(X)$ is a Lie group, we write $G^{0}$ for the connected component of $G$ containing the identity. By $\mathfrak{g}$ we denote 
the Lie algebra of $G$. If $D \subset X$ and $g$ is a metric on $X \backslash D$, then we define

$$
\operatorname{Aut}(X, D):=\left\{\phi \in \operatorname{Aut}(X)|\phi|_{D} \in \operatorname{Aut}(D)\right\}
$$

and

$$
\operatorname{Aut}(X, D, g):=\left\{\phi \in \operatorname{Aut}(X, D) \mid \phi^{*} g=g\right\} .
$$

If $Y$ is some complex manifold and $g$ a metric on $Y$, we also denote

$$
\operatorname{Aut}(Y, g):=\left\{\phi \in \operatorname{Aut}(Y) \mid \phi^{*} g=g\right\} .
$$

In most cases we will further assume that $X$ is almost homogeneous. We will often make use of the following equivalences.

Definition + Lemma 1.1. A compact complex manifold $X$ is called almost homogeneous, if there is a Lie group $G \subset \operatorname{Aut}(X)$ such that one (and then all) of the following properties are satisfied:

(i) The action of $G$ has an open orbit,

(ii) the action of $G^{0}$ has an open orbit,

(iii) $\mathfrak{g}:=T_{1} G$ generates $T_{X}$ at the general point,

(iv) there is a vector space $V \subset \mathfrak{g}$ with $\operatorname{dim} V=\operatorname{dim} X$, which generates $T_{X}$ at the general point.

If $G \subset \operatorname{Aut}^{0}(X)$ is a Lie group which has an open orbit, then we say $G$ acts almost transitively on $X$.

In the Kähler case we will encounter a special form of abelian Lie groups, so called semi-tori.

Definition 1.2. A complex Lie group $G$ is a semi-torus, if there is a number $n$ and a discrete subgroup $\Lambda \subset \mathbb{C}^{n}$ such that $\Lambda \otimes \mathbb{C}=\mathbb{C}^{n}$ and $G=\mathbb{C}^{n} / \Lambda$.

The other aspect of the paper is Kähler metrics on complements of divisors. If $Y$ is any complex manifold and $G \subset \operatorname{Aut}(Y)$ is a Lie group, we denote by $M_{G}(Y)$ the set of all Kähler metrics on $Y$ invariant under $G$. If $X$ is a compact complex manifold, $D \subset X$ a divisor, $Y=X \backslash D$ and $G \subset \operatorname{Aut}(X, D)$, then we also write $M_{G}(X, D):=M_{G}(Y)$ to emphasize that we use only automorphisms of $X$ for the construction.

For analytical application a Kähler potential is more useful than a $\bar{\partial}$-exact $(1,1)$ form. In the compact case there is no difference, but in the open case we have to distinguish. This is the reason why we do not consider the Kähler cone as a subcone of $H^{1,1}(X)$, but use a finer equivalence relation. 
Definition 1.3. For closed $(1,1)$-forms $\omega, \omega^{\prime}$ on a complex manifold $Y$,

$$
\omega \sim \omega^{\prime}: \Longleftrightarrow \text { there exists } \phi \in C^{\infty}(Y, \mathbb{R}) \text { such that } \omega-\omega^{\prime}=i \partial \bar{\partial} \phi,
$$

is an equivalence relation. We denote the quotient by $\mathscr{H}^{1,1}(Y)$. If $G \subset \operatorname{Aut}(Y)$, we define

$$
K_{G}(Y):=M_{G}(Y) / \sim \subset \mathscr{H}^{1,1}(Y)
$$

and call it the $G$-Kähler cone of $X \backslash D$. We also abbreviate $K(Y):=K_{1}(Y)$ and call this cone the Kähler cone of $Y$. Note that this definition of the Kähler cone coincides with the usual, if $Y$ is compact. Like above, if $X$ is a compact complex manifold, $D \subset X$ a divisor, $Y=X \backslash D$ and $G \subset \operatorname{Aut}(X, D)$, we also write $K_{G}(X, D):=K_{G}(Y)$.

Since parts of the paper are written in the language of differential geometry, we use co- and contravariant indexing conventions as well as Einstein's sum convention. In order to be able to do this, we distinguish indices arising from non-differential context by setting them in brackets, if appropriate. The decision, whether such an index is sub- or superscript, is made by considering the beauty of involved formulae. For example, a set of vector fields is denoted by $s^{(i)}$. The same vector fields in local coordinates will be written $s^{i k} \frac{\partial}{\partial z^{k}}$. Here we omit the brackets, because we want to put the components into a matrix. The only unlucky point of this convention is where powers of coordinates appear. But we believe that also in these cases the meaning will become clear.

\section{Metrics generated by vector fields}

2.1. Construction of the metrics. In this section we discuss a method to construct complete, Ricci-flat hermitian metrics. These are neither necessarily invariant nor necessarily Kähler. We will determine the cases when the metric is Kähler.

The construction of the divisor is widely used in many works about almost homogeneous manifolds. However, in this place we concentrate on the metric which comes with the construction. For this reason we give here a detailed description.

We use the notion of an abelian subspace of $H^{0}\left(T_{X} \otimes \mathcal{M}_{X}\right)$. We call $V \subset$ $H^{0}\left(T_{X} \otimes \mathcal{M}_{X}\right)$ abelian, if for all $\zeta, \xi \in V$ holds $[\zeta, \xi]=0$. In general, however, we do not require $V$ to be an algebra.

Construction 2.1. Let $X$ be a projective manifold of dimension $n, E$ an effective divisor and $\mathscr{B}=\left\{s^{(1)}, \ldots, s^{(n)}\right\} \subset H^{0}\left(T_{X} \otimes \mathcal{O}(E)\right)$ meromorphic vector fields generating $T_{X}$ in the general point and denote $V=\langle\mathscr{B}\rangle$ the vector space spanned by $\mathcal{B}$. This yields a divisor $D_{V} \in\left|-K_{X}+n E\right|$ and a Ricci-flat hermitian metric $g_{\mathcal{B}}$ on $X \backslash\left(D_{V} \cup E\right)$. The metric $g_{B}$ is Kähler if and only if $V$ is abelian. 
Implementation. Since $s^{(1)}, \ldots, s^{(n)} \in H^{0}\left(T_{X} \otimes \mathcal{O}(E)\right)$ generate $T_{X}$ in a general point, $\bigwedge_{i=1}^{n} s^{(i)}$ vanishes exactly on a divisor $D_{V}$. Obviously $D_{V} \in\left|-K_{X}+n E\right|$. Since on $X \backslash\left(D_{V} \cup E\right)$ the $s^{(i)}(x)$ form a basis of $T_{X, x}$, we may construct $s_{(i)} \in$ $\bar{T}_{X, x}^{*}$ by prescribing $s_{(i)}\left(\overline{s^{(j)}}\right)=\delta_{i j}$ on $X \backslash\left(D_{V} \cup E\right)$. Further we can extend this correspondence to a linear map

$$
{ }^{\dagger}: T_{X} \longrightarrow \bar{T}_{X}^{*}
$$

and define

$$
g_{\mathcal{B}}(s \otimes t):=s^{\dagger}(t)
$$

if $s \in T_{X, x}, t \in \bar{T}_{X, x}$. In a local chart we denote $s^{(i)}=s^{i k} \frac{\partial}{\partial z^{k}}$. We denote by $\left(s_{i j}\right)=\sigma$ the inverse matrix of $\left(s^{i j}\right)$. Then

$$
g_{\mathcal{B}, i j}=g_{\mathcal{B}}\left(\frac{\partial}{\partial z^{i}} \otimes \frac{\partial}{\partial \overline{z^{j}}}\right)=s_{i k} \overline{s_{j k}},
$$

which yields

$$
\operatorname{Ric}\left(g_{\mathcal{B}}\right)=\frac{i}{2 \pi} \partial \bar{\partial} \log \operatorname{det} g_{\mathcal{B}}=\frac{i}{2 \pi} \partial \bar{\partial} \log \operatorname{det} \sigma+\frac{i}{2 \pi} \partial \bar{\partial} \log \overline{\operatorname{det} \sigma}=0,
$$

since $\operatorname{det} \sigma$ is holomorphic.

A short calculation shows that $g_{\mathcal{B}}$ is Kähler if and only if

$$
s_{i j, l}=s_{l j, i}
$$

for all $i, j, l$. Converting this condition to the vector field components yields

$$
s^{i j} s_{, j}^{k l}=s^{k j} s_{, j}^{i l}
$$

for all $i, k, l$. Of course, this is the condition

$$
\left[s^{(i)}, s^{(k)}\right]=0
$$

for all $i, k$. But this means that $V$ is abelian.

Serre's Theorems A and B for projective manifolds imply that the conditions of the construction can be satisfied for any projective $X$, if we choose $E$ ample enough. Hence there is a Ricci-flat hermitian metric on the complement of an appropriate divisor for any projective $X$.

Note that by construction $T_{X \backslash\left(D_{V} \cup E\right)}$ is trivial. In [Wi04] the problem is addressed when $T_{X}(-\log D)$ is trivial and answered in terms of the existence and action of a semi-torus. In the next section we will be able to describe this property in terms of $g_{\mathscr{B}}$ and $D_{V}$. 
In the remainder of the article we will set $E=0$. This has two reasons. The exponential map, applied to meromorphic vector fields does not yield automorphisms of the whole manifold, hence there is no Lie group we can work with. The other reason is that we cannot expect completeness of the metric in direction to $E$. After setting $E=0$, we can drop the condition of projectiveness of $X$. On the other hand, the construction then exhibits $X$ as an almost homogeneous manifold.

2.2. Completeness of the metrics. Since we are dealing with open manifolds we should address the problem of completeness of the constructed metric. For this purpose we introduce some new notation. First, we define $S:=\left(s^{i k}\right)=\sigma^{-1}$. Recall that we chose $E=0$. We interpret $S^{t}: \mathcal{O}_{X}^{\oplus n} \rightarrow T_{X}$ as a sheaf homomorphism and define

$$
\mathcal{L}:=\operatorname{ker} S^{t}
$$

Of course, $\mathcal{L}$ is supported on $D_{V}$ and we prove

Lemma 2.2. If $D_{V}$ is smooth, then $\mathcal{L}$ is a line bundle on $D_{V}$.

Proof. $\mathcal{L}$ is line bundle if and only if $\mathrm{rk} S \mid D_{V}=n-1$ everywhere. So assume that in $x \in D_{V}$ we have rk $S(x)<n-1$. Then all $(n-1) \times(n-1)$-minors of $S$ vanish in $x$, in particular $d \operatorname{det} S(x)=0$, hence $x \in \operatorname{Sing}\left(D_{V}\right)$.

Now assume that $D_{V}$ is smooth and consider

$$
\omega_{(i)}:=\left.\bigwedge_{j \neq i} s^{(j)}\right|_{D_{V}} \in H^{0}\left(\bigwedge^{n-1} T_{X} \mid D_{V}\right)=H^{0}\left(\Omega_{X}^{1} \mid D_{V} \otimes N_{D_{V} \mid X}\right) .
$$

These are related via $\mathcal{L}$ by the equation

$$
(-1)^{j} \lambda_{(j)} \omega_{(i)}+(-1)^{i} \lambda_{(i)} \omega_{(j)}=0
$$

for all $i, j$ and $x \in D_{V}, \lambda \in \mathscr{L}_{x}$. Again, smoothness of $D_{V}$ implies that in a point $x \in D_{V}$ not all $\omega_{(i)}$ can vanish. Hence the vector spaces $\mathcal{F}_{x}:=\left\langle\left(\omega_{(i)}\right)_{i}\right\rangle$ are onedimensional and form a line bundle $\mathcal{F} \subset \Omega_{X}^{1} \mid D_{V} \otimes N_{D_{V} \mid X}$. By looking at the natural local trivializations of $\mathcal{L}$ and $\mathscr{F}$ it is easy to see that

$$
\mathcal{F} \cong \mathcal{L}^{\vee}
$$

Note that the inclusion $i: D_{V} \rightarrow X$ yields via Poincaré Duality a homomorphism $i_{*}: H^{*}\left(D_{V}, \mathbb{R}\right) \rightarrow H^{*}(X, \mathbb{R})$ of degree 2 . With this notation in mind, the very definition of $\mathcal{F}$ implies that

$$
i_{*} c_{1}(\mathcal{F})=c_{2}(X) .
$$


The tensored dual tangent sequence

$$
0 \longrightarrow \mathcal{O}_{D_{V}} \longrightarrow \Omega_{X}^{1} \mid D_{V} \otimes N_{D_{V} \mid X} \stackrel{\pi}{\longrightarrow} \Omega_{D_{V}}^{1} \otimes N_{D_{V} \mid X} \longrightarrow 0,
$$

allows us to formulate the property $\mathcal{F}=\mathcal{O}_{D_{V}}=\operatorname{ker} \pi$.

Lemma 2.3. If $D_{V}$ is smooth, then $g_{\mathscr{B}}$ is complete if and only if $\mathcal{F}=\operatorname{ker} \pi$.

Proof. Let us choose $x \in D_{V}$ and local coordinates in a small open subset $U \subset X$ such that $D_{V}=\left\{z^{1}=0\right\}$. Furthermore, denote $U^{\prime}:=U \cap D_{V}$ and pr: $U \rightarrow U^{\prime}$ the projection induced by the local coordinates. choose $0 \neq \lambda \in \mathcal{L}\left(U^{\prime}\right)$ and an order of $\mathscr{B}$ such that $\lambda_{(1)} \equiv 1$. If we now define $B$ by

$$
B_{i j}(z):= \begin{cases}1 & \text { if } i=j, \\ -\lambda_{(j)}(\operatorname{pr}(z)) & \text { if } i=1, j \neq 1, \\ 0 & \text { else }\end{cases}
$$

then $\tilde{S}:=B S$ is just $S$ replaced by a first row vanishing on $U^{\prime}$. Now we have a look at $\tilde{\sigma}:=(\tilde{S})^{-1}$. Since the first row is identically 0 on $U^{\prime}$, we conclude that $\tilde{s}_{1 i} \in \mathcal{O}(U)$ for $i>1$. Since

$$
g_{11}=\sum_{i>1}\left|\tilde{s}_{1 i}\right|^{2}-2 \operatorname{Re}\left(\overline{\tilde{s}_{11}} \sum_{i>1} \lambda_{(i)} \tilde{s}_{1 i}\right)+\left|\tilde{s}_{11}\right|^{2} \sum\left|\lambda_{(j)}\right|^{2},
$$

we see now that $g$ is complete if and only if $\tilde{s}_{11} \sim \frac{1}{z^{1}}$ for all such choices of coordinates. Indeed, $\tilde{s}_{11}=s_{11}$. Hence, if we denote $A_{i j}$ the $(i, j)$-entry of the cofactor matrix of $S$, then the condition is equivalent to $A_{11}(x) \neq 0$. If we now choose other coordinates $z^{\prime 1}, \ldots, z^{\prime n}$ such that $D_{V}=\left\{z^{1}=0\right\}$ and denote $J:=\left(\frac{\partial z^{j}}{\partial z^{i}}\right)_{i j}, h:=\frac{z^{1}}{z^{1}} \neq 0$, then

$$
A_{11}^{\prime}=\operatorname{det} J\left(h^{-1} A_{11}+\sum_{k>1} J_{1 k}^{-1} A_{1 k}\right) \neq 0
$$

Since the coordinate transform was arbitrary, we conclude that

$$
A_{1 k}(x)=0 \quad \text { for all } k>1 .
$$

This is equivalent to $0 \neq \omega_{(1)} \in H^{0}(\operatorname{ker} \pi)$. This again means $\mathcal{F}=\operatorname{ker} \pi$.

Note that completeness as well as the Kähler property of $g_{\mathscr{B}}$ depend only on $V$.

If $D_{V}$ is not smooth, but still reduced, then we consider $D_{V}^{0}$, the regular part of $D_{V}$ and the corresponding objects $\mathcal{F}^{0}, \mathcal{L}^{0}, \pi^{0}$, which are obtained by restriction to $D_{V}^{0}$. We now show the preceding lemma for the singular case. 
Lemma 2.4. Assume that $D_{V}$ is reduced. Then $g_{\mathcal{B}}$ is complete if and only if $\mathscr{F}^{0}=$ $\operatorname{ker} \pi^{0}$.

Proof. ' $\Rightarrow$ ': If $g_{\mathscr{B}}$ is complete, the same arguments as in Lemma 2.3 imply that $\mathcal{F}^{0}=\operatorname{ker} \pi^{0}$.

' $\Leftarrow$ ': Let locally $D_{V}=\{f=0\}$ in a small open neighbourhood $U \subset X$. Then we can choose functions $z^{2}, \ldots z^{n}$ which give local coordinates together with $f$ on the set $\tilde{U}:=U \backslash\left\{d f \wedge d z^{2} \wedge \cdots \wedge d z^{n}=0\right\}$. Like in the proof above we argue that $g_{\mathscr{B}}$ is complete if $A_{11}(x) \neq 0$ for choices like above and $x \in D_{V}$. Since by assumption this is true for $x \in D_{V} \backslash \operatorname{Sing}\left(D_{V}\right)$, extension of the holomorphic function $A_{11}$ to $U^{\prime}$ yields a non-zero function $A_{11} \in \mathcal{O}^{*}\left(U^{\prime}\right)$, if $D_{V}$ was normal. If $D_{V}$ is not normal, we choose an embedded normalization

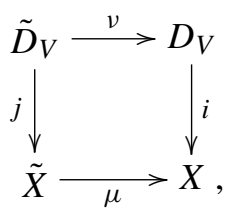

where $i, j$ denote inclusions and $v$ the normalization of $D_{V}$. Now we apply the same arguments to the pseudometric $\mu^{*} g$ and obtain by looking at paths $\gamma$ such that $\left.\mu\right|_{\gamma}$ is a diffeomorphism that $g$ is complete.

Now we can see a connection to the invariance group.

Lemma 2.5. Assume that $D_{V}$ is reduced. Then $g_{\mathscr{B}}$ is complete if and only if $\exp (V) \subset$ $\operatorname{Aut}^{0}\left(X, D_{V}\right)$.

Proof. If we denote by $\mathcal{V}$ the sheaf on $D$ generated by $\mathcal{V}_{x}:=\left\langle\left\{s^{(i)}(x)\right\}\right\rangle$ for $x \in D$, then $\mathcal{F}(\mathcal{V}) \equiv 0$, if we regard $\mathcal{F} \subset \operatorname{Hom}\left(\left.T_{X}\right|_{D_{V}}, N_{D_{V} \mid X}\right)$. Since $\mathcal{F}^{0}=\operatorname{ker} \pi^{0}$, figuring out the dualized maps

$$
0 \longrightarrow T_{D_{V}^{0}} \longrightarrow T_{X} \mid D_{V}^{0} \stackrel{p^{0}}{\longrightarrow} \mathcal{F}^{0 \vee} \otimes N_{D_{V}^{0} \mid X} \longrightarrow 0
$$

yields $\mathcal{V}^{0}=\operatorname{ker} p^{0}=T_{D_{V}^{0}}$. This is equivalent to $\left.V\right|_{D_{V}^{0}} \subset H^{0}\left(T_{D_{V}^{0}}\right)$. This again means that every $\phi \in \exp (V)$ holds $D_{V}$ invariant, so $\exp (V) \subset \operatorname{Aut}^{0}\left(X, D_{V}\right)$ is an equivalent condition.

Taking into account the results of Section 3, which are obtained independently of the considerations about completeness, we even find

Theorem 2.6. If $V$ is a Lie subalgebra, then $g_{\mathscr{B}}$ is complete. If $g_{\mathscr{B}}$ is complete and $D_{V}$ is reduced, then $V$ is a Lie subalgebra. 
Proof. (i) If $V$ is a Lie subalgebra, Lemma 3.1 implies the desired property $\exp (V) \subset$ $\operatorname{Aut}^{0}\left(X, D_{V}\right)$. Here we are finished, if $D_{V}$ is reduced. In any case, if we have $D_{V, \text { red }} \cap U=\left\{z^{1}=0\right\}$, this implies that

$$
s^{i 1}=z^{1} t^{i 1}
$$

for some $t^{i 1} \in \mathcal{O}(U)$ and all $i$. Now

$$
\operatorname{det} S=\sum_{i} s^{i 1} A_{1 i}=z^{1} \sum_{i} t^{i 1} A_{1 i} .
$$

So, if $m:=\max \left\{k \mid\left(z^{1}\right)^{-k} A_{1 i} \in \mathcal{O}(U)\right.$ for all $\left.i\right\}$, we conclude $\left(z^{1}\right)^{-m-1} \operatorname{det} S \in$ $\mathcal{O}(U)$. Hence

$$
g_{11}=\sum\left|s_{1 i}\right|^{2}=(\operatorname{det} S)^{-2} \sum\left|A_{1 i}\right|^{2} \sim\left|z^{1}\right|^{-k}
$$

with $k \geq 2$. This procedure generalizes easily to the case of $D_{V \text {,red }}$ being normal crossings. But this we can achieve by Hironaka's embedded desingularization. Since $\operatorname{Sing}\left(D_{V \text {,red }}\right)$ is also $\exp (V)$-invariant and so is $\operatorname{Sing}\left(\operatorname{Sing}\left(D_{V, \text { red }}\right)_{\text {red }}\right)$ and so on, we can pull back the vector fields to the normal crossings case and apply the arguments above.

(ii) Since $g_{\mathscr{B}}$ is complete and $D_{V}$ reduced, by Lemma 2.5 we obtain $\exp (V) \subset$ $\operatorname{Aut}^{0}\left(X, D_{V}\right)$. Hence $\operatorname{Aut}^{0}\left(X, D_{V}\right)$ acts almost transitively. So Lemma 3.2 yields $\operatorname{dim} \exp (V)=\operatorname{dim} X=\operatorname{dim}_{\operatorname{Aut}^{0}}\left(X, D_{V}\right)$. This means

$$
T_{1} \operatorname{Aut}^{0}\left(X, D_{V}\right)=T_{1} \exp (V)=V,
$$

hence $V$ is a Lie subalgebra.

Now it is clear from the previous arguments that $T_{X}\left(-\log D_{V}\right)$ is trivial, if $g_{\mathcal{B}}$ is complete and $D_{V}$ is a simple normal crossings divisor. In Chapter 4 we will show furthermore that $G:=\exp (V)$ is a semi-torus, if $g_{\mathscr{B}}$ is complete and Kähler and $D_{V}$ is reduced.

The existence of a complete $g_{\mathscr{B}}$ for a smooth $D_{V}$ restricts the geometry of $X$ significantly:

Corollary 2.7. If $D_{V}$ is smooth and $g_{\mathscr{B}}$ is complete, then $c_{2}(X)=0$.

Proof. $\mathcal{F}=\operatorname{ker} \pi \cong \mathcal{O}_{D_{V}}$, hence $c_{2}(X)=i_{*} c_{1}\left(\mathcal{O}_{D_{V}}\right)=0$.

Now we have seen that $c_{2}(X) \neq 0$ implies that the divisor $D_{V}$ is singular, if $V$ is a Lie subalgebra. We will see later that the Kähler condition allows an explicit description of the singularities, at least on projective homogeneous manifolds. 


\section{Symmetries of the divisor and the metric}

In this section we want to relate the construction above (with $E=0$ ) to the appearance of symmetries on $D$ and the metric. As it may be not hard to guess, this connection is made by Lie theory. Throughout this section we mean always a complex Lie group when we speak of Lie groups.

If $G$ is a Lie group we identify $\mathfrak{g}=T_{1} G$, and if $G \subset \operatorname{Aut}^{0}(X)$, then we furthermore identify $\mathfrak{g}$ with the subvector space of $H^{0}\left(T_{X}\right)$ given by the vector fields $s(x):=$ $\left.\frac{\partial}{\partial t} g(t) x\right|_{t=0}$, where $g(t)$ denotes a (holomorphic) path in $G$ with $g(0)=1$ and $\left.\frac{\partial}{\partial t} g(t)\right|_{t=0}=\xi \in T_{1} G$. Furthermore we have an action of $G$ on $T_{1} G$ by $h \xi:=$ $\left.\frac{\partial}{\partial t} h g(t) h^{-1}\right|_{t=0}$, if $h \in G$.

Lemma 3.1. Let $X$ be a compact complex manifold of dimension $n, G \subset \operatorname{Aut}^{0}(X)$ a connected complex Lie group acting almost transitively on $X$ and $\mathfrak{g}$ be the corresponding Lie algebra. Then

(i) $D \in\left|-K_{X}\right|$ is reduced and $G \subset \operatorname{Aut}^{0}(X, D) \Rightarrow D=D_{V}$ for all $V \subset \mathfrak{g}$ with $\operatorname{dim} V=n$ and generating $T_{X}$ in the general point,

(ii) if $\operatorname{dim} G=n$, then $G \subset \operatorname{Aut}^{0}\left(X, D_{\mathfrak{g}}\right)$,

(iii) if $\operatorname{dim} G=n, \mathscr{B} \subset \mathfrak{g}$ is a basis, then

$$
G \subset \operatorname{Aut}^{0}\left(X, D_{\mathfrak{g}}, g_{\mathscr{B}}\right) \Longleftrightarrow G \text { is abelian. }
$$

Proof. (i): Let $V \subset \mathfrak{g}$ be an $n$-dimensional vector space generating $T_{X}$ in the general point and $\mathcal{B} \subset V$ a basis. Since $D$ is $G$-invariant, for any $s \in \mathfrak{g} \subset H^{0}\left(T_{X}\right)$ the restriction $\left.s\right|_{D^{0}}$ gives an element of $H^{0}\left(T_{D^{0}}\right)$. Since $\operatorname{dim} D=n-1$, this implies $\left.\bigwedge_{s \in \mathcal{B}} s\right|_{D}=0$, hence $D \subset D_{V}$. But $D$ and $D_{V}$ are both elements of $\left|-K_{X}\right|$, hence $D=D_{V}$.

(ii): If $s \in \mathfrak{g} \subset H^{0}\left(T_{X}\right)$ is given by $\xi \in T_{1} G$, then for $h \in G$ the pullback $h^{*} s$ is given by $h^{-1} \xi \in T_{1} G$, hence $h^{*} s \in \mathfrak{g}$. Furthermore $h^{*}$ maps a basis of $\mathfrak{g}$ to a basis again, because $h$ is an automorphism. This proves that $\bigwedge s^{(i)}=0 \Longleftrightarrow$ $\bigwedge h^{*} s^{(i)}=0$, if $s^{(1)}, \ldots, s^{(n)}$ is a basis of $\mathfrak{g}$. Hence $h(D)=D$. This proves that $D$ is $G$-invariant.

(iii): Let $\omega$ be the fundamental $(1,1)$-form of $g_{\mathcal{B}}$. Of course, $g_{\mathcal{B}}$ is $G$-invariant if and only if $\mathscr{L}_{s} \omega=0$, if $s \in \mathfrak{g}$ and $\mathcal{L}$ denotes the Lie derivative. If $C_{s}$ denotes the contraction by $s$, then $\mathcal{L}_{s}=d C_{s}+C_{s} d$ (see e.g. [La, V,5]). Let us choose local coordinates like in the construction. Then

$$
0=\mathcal{L}_{s^{(l)}} \omega=\sum_{i, j, k, m} s^{l m}\left(s_{i k, m}-s_{m k, i}\right) \bar{s}_{j k} d z^{i} \wedge d \bar{z}^{j} .
$$

Since $S$ is invertible on $X \backslash D$, we obtain

$$
s_{i k, m}-s_{m k, i}=0
$$


for all $i, k, m$. This we identified at an earlier place with the condition

$$
\left[s^{(i)}, s^{(j)}\right]=0
$$

for all $i, j$. Hence $\mathfrak{g}$ is abelian. It is well known that this is equivalent to $G$ to be abelian.

As a first application, we obtain kind of uniqueness of the vector space $V$ in the construction.

Lemma 3.2. Let $X$ be a compact complex manifold and $D \in\left|-K_{X}\right|$ reduced. If $\operatorname{Aut}(X, D)$ acts almost transitively, then $\operatorname{dim} \operatorname{Aut}(X, D)=\operatorname{dim} X$.

Proof. Set $G:=\operatorname{Aut}(X, D)$ and $n:=\operatorname{dim} X$. Of course, $\operatorname{dim} G \geq n$, since otherwise $T_{1} G$ could not generate $T_{X}$ in any point. Now choose $\mathbb{B}:=\left\{s^{(1)}, \ldots, s^{(n+1)}\right\} \subset \mathfrak{g}$ such that $\mathscr{B} \backslash\left\{s^{(n+1)}\right\}$ generates $T_{X}$ in the general point. Further denote $\eta_{(i)}:=$ $\bigwedge_{j \neq i} s^{(j)}$ and by $V_{(i)}$ the vector space generated by $\mathscr{B} \backslash\left\{s^{(i)}\right\}$.

Since $\operatorname{dim} X=n$, we can find meromorphic functions $f_{(i)} \in \mathcal{M}_{X}(X)$ such that $s^{(n+1)}=\sum_{i=1}^{n} f_{(i)} s^{(i)}$. By assumption we know $\eta_{(n+1)} \not \equiv 0$.

If $\eta_{(i)} \equiv 0$, then we see by $\eta_{(i)}=f_{(i)} \eta_{(n+1)}$ that $f_{(i)} \equiv 0$.

If $\eta_{(i)} \not \equiv 0$, then $V_{(i)}$ generates $T_{X}$ in the general point and we may use Lemma 3.1 to obtain

$$
\eta_{(n+1)}=0 \Longleftrightarrow \eta_{(i)}=0 \Longleftrightarrow f_{(i)} \eta_{(n+1)}=0 .
$$

Hence $f_{(i)}$ has no zeroes. By exchanging $s^{(i)}$ and $s^{(n+1)}$ we also see that $f_{(i)}$ has no poles. Hence $f_{(i)}$ is constant.

Now we proved that every $f_{i}$ is constant, hence $s^{(1)}, \ldots, s^{(n+1)}$ are linearly dependent and $\operatorname{dim} G=n$.

Note that the connection between the invariance group and the anticanonical system is essential. For example, the invariance group of a point in $\mathbb{P}^{1}$ is twodimensional and acts almost transitively.

Since every $\operatorname{dim} X$-dimensional Lie group which acts almost transitively yields an invariant $D \in\left|-K_{X}\right|$, Lemma 3.2 suggests the following definition.

Definition 3.3. If $X$ is a compact complex manifold and $G \subset \operatorname{Aut}(X)$ a connected complex Lie group with $\operatorname{dim} G=\operatorname{dim} X$ acting almost transitively on $X$, we say $G$ is a divisorial group. If on the other hand $D \in\left|-K_{X}\right|$ is reduced, we say $D$ has a divisorial invariance group, if $\operatorname{Aut}(X, D)$ acts almost transitively. Any object invariant under a divisorial group we call divisorially invariant.

Lemma 3.2 now allows a stronger and more compact formulation of Lemma 3.1. 
Theorem 3.4. Let $X$ be a compact complex manifold, $G$ a divisorial group and $\mathfrak{g}$ the corresponding Lie algebra. Then for a reduced divisor $D \in\left|-K_{X}\right|$ holds

$$
G=\operatorname{Aut}^{0}(X, D) \Longleftrightarrow D=D_{\mathfrak{g}} .
$$

If $\mathscr{B} \subset \mathfrak{g}$ is a basis, then

$$
G=\operatorname{Aut}^{0}\left(X, D_{\mathfrak{g}}, g_{\mathscr{B}}\right) \Longleftrightarrow G \text { is abelian. }
$$

Proof. If $D=D_{\mathfrak{g}}$, by Lemma $3.1 G \subset \operatorname{Aut}^{0}(X, D)$. Hence $\operatorname{Aut}^{0}(X, D)$ acts almost transitively and by Lemma 3.2 we obtain $\operatorname{dim} G=\operatorname{dim} X=\operatorname{dim}_{\operatorname{Aut}^{0}}(X, D)$, hence $G=\operatorname{Aut}^{0}(X, D)$.

In this context it is appropriate to introduce the notion of a homogeneous pair.

Definition 3.5. A homogeneous pair $(X, D)$ consists of a compact complex manifold $X$ and an effective reduced divisor $D$ such that $\operatorname{Aut}^{0}(X, D)$ acts transitively on $X \backslash D$. We call a homogeneous pair $(X, D)$ anticanonical, if $D \in\left|-K_{X}\right|$.

Theorem 3.4 provides a close relation between homogeneous pairs and divisorial groups.

\section{Remark 3.6.}

- Note that the proof of Lemma 3.1 also shows that every analytical $G$-invariant set $S$ is contained in the $G$-invariant $D \in\left|-K_{X}\right|$.

- Note that the vector field method is much more general than the invariance approach: There is no need for the vector space $V \subset H^{0}\left(T_{X}\right)$ to be an algebra, whereas invariant divisors correspond to Lie subalgebras of $H^{0}\left(T_{X}\right)$.

- However, if $g$ arises by the general vector field method and is Kähler, we have $V$ proved to be abelian; in particular, $V$ is a Lie subalgebra. Of course, exp: $H^{0}\left(T_{X}\right) \rightarrow \operatorname{Aut}^{0}(X)$ restricted to $V$ maps to an $n$-dimensional Lie subgroup $G$ leaving $D$ and $g$ invariant.

Now we also see that divisorial invariance is exactly the property we had in mind when we expected that Ricci-flatness should be implied by a high order of symmetry.

Corollary 3.7. Let $X$ be an $n$-dimensional compact complex manifold, $G \subset \operatorname{Aut}^{0}(X)$ a divisorial (abelian) Lie group. Then there is a complete Ricci-flat (Kählerian $G$-invariant) metric on $X \backslash D_{\mathfrak{g}}$.

In [Wi04] Winkelmann proved that $T_{X}(-\log D)$ is even holomorphically trivial, if $G$ is a complex semi-torus and acts with only semi-tori as isotropy groups. We will see in the next paragraph that $G$ being a semi-torus is implied by $D_{\mathfrak{g}}$ being reduced. 


\section{The structure of the Kähler case}

4.1. Description of the manifolds. We saw that the metric $g$ on $X \backslash D$ constructed by an automorphism group $G$ is complete and it is Kähler if and only if $G$ is abelian. In this case $g$ is also $G$-invariant. Now we want so see that this construction yields all $G$-invariant Kähler metrics. The first step is to show that complex invariance groups of Kähler metrics are abelian. Similar connections between the Kähler property and abelian groups are well known. However, most results in this direction use compactness of the Kähler manifold by employing that all holomorphic one-forms are closed. This formulation makes only use of the Kähler form and hence is also valid in the non-compact case.

Lemma 4.1. Let $Y$ be a complex manifold and $g$ a Kähler metric on $Y$. Then any connected complex Lie group $G \subset \operatorname{Aut}^{0}(Y, g)$ is abelian.

Proof. Let $\omega$ denote the Kähler form of $g$ and $\mathfrak{g} \subset H^{0}\left(T_{Y}\right)$ the Lie algebra of $G$. Since $g$ is $G$-invariant, for all $s \in \mathfrak{g}$ we obtain

$$
\mathcal{L}_{S} \omega=0,
$$

where $\mathcal{L}$ denotes the Lie derivative. If furthermore $C$ denotes the contraction by the subscript vector field, $\mathscr{L}_{s}=d C_{s}+C_{s} d$ and hence we conclude $d C_{s} \omega=0$ for all $s \in \mathfrak{g}$. Again using an elementary formula (see e.g. [La, V,5]) and $d \omega=0$ we obtain for $s, t \in \mathfrak{g}$

$$
C_{[s, t]} \omega=\left(\mathscr{L}_{s} C_{t}-C_{t} \mathcal{L}_{s}\right) \omega=\mathscr{L}_{s} C_{t} \omega=d C_{s} C_{t} \omega+C_{s} d C_{t} \omega .
$$

Since $\omega$ is a $(1,1)$-form and $s, t$ are holomorphic, $C_{s} C_{t} \omega=0$. We already saw that $d C_{t} \omega=0$, hence both summands of the right hand side vanish, yielding $C_{[s, t]} \omega=0$. In local coordinates this means

$$
g_{\alpha \bar{\beta}}[s, t]^{\alpha}=0 .
$$

Since the matrix $g_{\alpha \bar{\beta}}$ is invertible this implies $[s, t]=0$. Hence $\mathfrak{g}$ is abelian and therefore also $G$ is abelian.

It is known (cf, [On1, p. 12]) that an orbit of $G$ is locally (in $G$ ) a submanifold. A closer look at the argument in the second part of the proof of Theorem 3.4 reveals that any orbit, whose local dimension is smaller than $n$ must be contained in $D_{\mathfrak{g}}$. Hence $X \backslash D_{\mathfrak{g}}$ is the unique open orbit of $G$. Let $x_{0} \in X \backslash D_{\mathfrak{g}}$ and $\alpha: G \rightarrow X \backslash D_{\mathfrak{g}}$ be the action map $g \mapsto g x_{0}$. Again it is known (cf. [On1]) that $\alpha$ has constant rank. Since $\alpha$ is surjective, it has to be a covering map. If $G$ is abelian, $y z:=\alpha\left(\alpha^{-1}(y) \alpha^{-1}(z)\right)$ is well-defined and turns $X \backslash D_{\mathfrak{g}}$ itself into an abelian Lie group of dimension $n$ and $\alpha$ 
into a group homomorphism. Since elements of $\operatorname{ker} \alpha$ induce the same action on $X \backslash D_{\mathfrak{g}}$, the property $G \subset \operatorname{Aut}^{0}(X)$ implies that $\alpha$ is an isomorphism. Hence we will identify $G$ and $X \backslash D_{\mathfrak{g}}$ from now on.

Note that for a Lie group $G$ being abelian implies that $G=\mathbb{C}^{n} / \Lambda$, where $\Lambda$ is a discrete subgroup. This is proved by looking at the exponential map

$$
\exp : \mathfrak{g} \longrightarrow G
$$

which is easily seen to be a group homomorphism of $(\mathfrak{g},+)$ into $G$. Since exp maps some neighbourhood of 0 diffeomorphically to a neighbourhood of 1 , say $U$, and $\bigcup_{k=1}^{\infty} U^{k}=G$, the map exp is surjective. Hence $G=(\mathfrak{g},+) / \operatorname{ker}(\exp )=\mathbb{C}^{n} / \Lambda$, where $\Lambda:=\operatorname{ker}(\exp )$ must be discrete, since $n=\operatorname{dim} G=\operatorname{dim} \mathfrak{g}$.

In particular we see that $X \backslash D_{\mathfrak{g}}=\mathbb{C}^{n} / \Lambda$, if $G$ is abelian. We will use this in the next proof.

Lemma 4.2. Let $X$ be a compact complex manifold and $G \subset \operatorname{Aut}^{0}(X)$ a divisorial Lie group. If $g$ is a Kähler metric on $X \backslash D_{\mathfrak{g}}$ such that $\operatorname{Aut}^{0}\left(X, D_{\mathfrak{g}}, g\right)=G$ then $g$ is complete and there is a basis $\mathcal{B} \subset \mathfrak{g}$ such that $g=g_{\mathscr{B}}$.

Proof. We already know by Lemma 4.1 that $G$ is abelian and hence $g_{\mathscr{B}}$ is Kähler and $G$-invariant, if $\mathscr{B} \subset \mathfrak{g}$ is a basis. Since $G=X \backslash D_{\mathfrak{g}}=\mathbb{C}^{n} / \Lambda$ we choose the images of the canonical coordinates $z_{1}, \ldots, z_{n}$ of $\mathbb{C}^{n}$ as local coordinates of $X \backslash D_{\mathfrak{g}}$. For the sake of simplicity we call them also $z_{1}, \ldots, z_{n}$. Of course, $g=g_{\alpha \bar{\beta}} d z^{\alpha} \otimes d \bar{z}^{\beta}$ is $G$-invariant, if and only if $g_{\alpha \bar{\beta}}$ is constant for all $\alpha, \beta$. Hence $g$ is complete and corresponds one to one to $g(0)$ what we identify with the matrix $g=\left(g_{\alpha \bar{\beta}}(0)\right)$. The corresponding matrix $\underline{g}_{\mathcal{B}}$ is $\underline{g}_{\mathcal{B}}=\sigma \sigma^{*}$. Note that $\sigma$ is constant since $G$ is abelian (cf. proof of Theorem 3.4). Recall $S=\sigma^{-1}$ and define $H:=S g S^{*}$. Since $H$ is hermitian, we can find $A \in \operatorname{Gl}(n)$ such that $H=A^{*} A$. Now $\underline{g}=\sigma \bar{H} \sigma^{*}=\sigma A A^{*} \sigma^{*}$. Set $B:=A^{-1}$. Then $g$ is given by the vector fields $t^{(i)}=\sum_{j} b_{i j} s^{(j)}$, which form another basis of $\mathfrak{g}$. (Indeed, this shows by Theorem 2.6 once more that $g$ is complete.)

Corollary 4.3. Let $X$ be a compact complex manifold and $S \subset X$ analytic with $\operatorname{codim} S>1$. If $X$ allows for a divisorially invariant Kähler metric on $X \backslash S$, then $X$ is a torus and $S$ is empty (if chosen minimal).

Proof. Assume that $g$ is such a metric and $G$ the divisorial abelian Lie group. By Lemma $4.2 \mathrm{~g} \mid X \backslash D_{\mathfrak{g}}$ is constructed by a basis of $\mathfrak{g}$. If $D_{\mathfrak{g}}$ is given by $\sigma \in H^{0}\left(-K_{X}\right)$, then $\operatorname{det} g=|\sigma|^{-2}$, hence is singular on $D_{\mathfrak{g}}$. This implies $D_{\mathfrak{g}}=0$. In particular, $X=G=\mathbb{C}^{n} / \Lambda$. Since $X$ is compact, $\Lambda$ is a complete lattice and $X$ is a torus.

Note that this proof works also, if $\operatorname{codim} S=1$, but $S \neq D_{\mathfrak{g} \text {,red. }}$. 
It is generally known (earliest references refer to a lecture of Reinhold Remmert in 1958/1959) that any abelian complex Lie group is a direct product of copies of $T$, $\mathbb{C}$ and $\mathbb{C}^{*}$, where $T$ is a group without non-constant holomorphic functions. For a more detailed analysis we refer to [Mo66]. Since Winkelmann related the triviality of a logarithmic tangent bundle to $G$ being a semi-torus in [Wi04], we would like to express this property in terms of the mentioned decomposition.

Lemma 4.4. $G=T \times\left(\mathbb{C}^{*}\right)^{k} \times \mathbb{C}^{l}$ is a semi-torus $\Longleftrightarrow l=0$.

Proof. If $G$ is not a semi-torus, i.e. $\Lambda \otimes \mathbb{C} \neq \mathbb{C}^{n}$, then obviously $l \neq 0$. So let $G$ be a semi-torus. There is a lattice $\Lambda^{\prime} \subset \mathbb{C}^{n-l}$ coming from the decomposition such that $G=\mathbb{C}^{n} / \Lambda^{\prime}$. The isomorphism $\tilde{\phi}: \mathbb{C}^{n} / \Lambda \rightarrow \mathbb{C}^{n} / \Lambda^{\prime}$ is induced by a vector space isomorphism $\phi: \mathbb{C}^{n} \rightarrow \mathbb{C}^{n}$ obeying $\phi(\Lambda)=\Lambda^{\prime}$. Hence $\mathbb{C}^{n-l}=\Lambda^{\prime} \otimes \mathbb{C}=$ $\phi(\Lambda \otimes \mathbb{C})=\mathbb{C}^{n}$, so $l=0$.

The toric varieties fit in the system as special cases where $G=\left(\mathbb{C}^{*}\right)^{n}$. If $X$ is Fano, then $X \backslash D_{\mathfrak{g}}$ is Stein and hence the factor $T$ does not occur (cf. also [MM60, Prop. 4] for this particular claim). If we relax this condition a little we can show that $T$ is a torus. The following lemma in particular covers the case of homogeneous manifolds for which the result is well known.

Lemma 4.5. Let $X$ be a projective almost homogeneous manifold such that $\left|-m K_{X}\right|$ is base point free for a certain $m>0$. Let further $G \subset \operatorname{Aut}^{0}(X)$ be an abelian divisorial Lie group. $X \backslash D_{\mathfrak{g}}$ is of the form $T \times \mathbb{C}^{k} \times\left(\mathbb{C}^{*}\right)^{l}$, where $T$ is a torus. Furthermore, $X=T \times Y$ for a rational manifold $Y$.

Proof. We first have to prove that $T$ is a torus. The assumption that $\left|-m K_{X}\right|$ is base point free enables us to choose for every $x \in D_{\mathfrak{g}}$ a meromorphic function $\tilde{f}$ with poles exactly along $D_{\mathfrak{g}}$ and $x$ is not in the locus of indeterminacy of $\tilde{f}$. If $T$ is not compact, we fix $z \in \mathbb{C}^{k} \times\left(\mathbb{C}^{*}\right)^{l}$ and $x \in \overline{T \times\{z\}}$. Since $\left.\tilde{f}\right|_{X \backslash D_{\mathfrak{g}}}$ is holomorphic, in particular $f:=\left.\tilde{f}\right|_{T \times\{z\}}$ is holomorphic. Hence $f$ is constant and we obtain $\overline{T \times\{z\}} \subset X \backslash D_{\mathfrak{g}}$. This implies that $T$ is compact.

Hence $T$ is a projective manifold. Since $T=\mathbb{C}^{k} / \Lambda$, the lattice $\Lambda$ is complete and hence $T$ is a torus.

Now we have to prove that the projection onto $T$ is extendable. Since $X$ is bimeromorphic to $\mathbb{P}^{n} \times T$, and the Albanese torus $A(X)$ as well as the Albanese map $\alpha: X \rightarrow A(X)$ are bimeromorphic invariants of projective manifolds, $T=A(X)$ and $\mathrm{pr}=\alpha$ by the universal property of $(A(X), \alpha)$.

Now choose $h:=\left(1, t^{\prime}\right) \in G=H \times T$ and denote $F_{t}:=\alpha^{-1}(t)$. Of course, $h: X \rightarrow X$ satisfies $h\left(F_{t}\right)=F_{t+t^{\prime}}$ and the map $\psi: F_{0} \times T \rightarrow X,(y, t) \mapsto(1, t) y$ is an isomorphism. Since $Y:=F_{0}$ is a compactification of $\mathbb{C}^{k} \times\left(\mathbb{C}^{*}\right)^{l}$, it is rational. 
Indeed, all factors of $G$ can occur. If $X$ factors $X=T \times \tilde{X}$, then $X \backslash D_{\mathfrak{g}}=$ $T \times \tilde{X} \backslash \tilde{D}$, since $K_{T}=0$. The factor $\mathbb{C}^{k} \times\left(\mathbb{C}^{*}\right)^{l}$ occurs even for $X=\mathbb{P}^{n}$, if $G$ is carefully chosen. For example, the group

$$
G=\left\{\left(\begin{array}{ccc}
1 & t & s+\frac{t^{2}}{2} \\
0 & 1 & t \\
0 & 0 & 1
\end{array}\right) \mid s, t \in \mathbb{C}\right\} \cong\left(\mathbb{C}^{2},+\right)
$$

acts on $\mathbb{P}^{2}$, leaving only $D_{\mathfrak{g} \text {,red }}=\left\{z^{2}=0\right\}$ invariant. Of course, the corresponding vector fields $s^{(1)}=z^{2} \frac{\partial}{\partial z^{0}}, s^{(2)}=z^{2} \frac{\partial}{\partial z^{1}}+z^{1} \frac{\partial}{\partial z^{0}}$ yield $D_{\mathfrak{g}}=\left\{\left(z^{2}\right)^{3}=0\right\}$. We leave it as an exercise to the reader to construct the other cases. The philosophy is: $\mathbb{C}^{*}$-actions degenerate to $\mathbb{C}$-actions whenever two hypersurfaces coincide. Indeed, we are now going to show that this is always the situation.

Lemma 4.6. If $D_{\mathfrak{g}}$ is reduced, then $G$ is a semi-torus.

Proof. We assume that $G$ is not a semi-torus, hence by Lemma 4.4 we obtain a decomposition $G=\mathbb{C} \times G^{\prime}$. We choose $s \in H^{0}\left(-K_{X}\right)$ such that $D_{\mathfrak{g}}=\{s=0\}$. Since $G^{\prime}$ is abelian, $G^{\prime}=\mathbb{C}^{n-1} / \Lambda$ and we may choose local coordinates $\tilde{z}^{1}, \ldots, \tilde{z}^{n-1}$ induced by canonical coordinates of $\mathbb{C}^{n-1}$. In those coordinates of $X \backslash D_{\mathfrak{g}}$ we write

$$
s=f\left(x, \tilde{z}^{1}, \ldots, \tilde{z}^{n-1}\right) \frac{\partial}{\partial x} \wedge \frac{\partial}{\partial \tilde{z}^{1}} \wedge \cdots \wedge \frac{\partial}{\partial \tilde{z}^{n-1}},
$$

where $x$ denotes the coordinate of the factor $\mathbb{C}$. If $x \rightarrow \infty$, we will approximate a point in $D_{\mathfrak{g}}$. In order to approximate other points (and indeed by this procedure all other points of a certain component of $D_{\mathfrak{g}}$ ), we choose an arbitrary holomorphic $\lambda: \mathbb{C} \rightarrow G^{\prime}$ and look at the curve $\left(x, \tilde{z}^{\prime}+\lambda(x)\right)$ for a fixed point $\tilde{z}^{\prime}:=\left(\tilde{z}^{1}, \ldots, \tilde{z}^{n-1}\right) \in G^{\prime}$. Let $p=\lim _{x \rightarrow \infty}\left(x, \tilde{z}^{\prime}+\lambda(x)\right) \in D_{\mathfrak{g}}$. By $x \mapsto \frac{1}{x}=: y, \tilde{z}^{i} \mapsto \tilde{z}^{i}-\lambda^{i}(x)=: z^{i}$ we get local coordinates in a neighbourhood $U(p) \backslash D_{\mathfrak{g}}$. In these coordinates,

$$
s=-f\left(\frac{1}{y}, z^{\prime}+\lambda\left(\frac{1}{y}\right)\right) y^{2} \frac{\partial}{\partial y} \wedge \frac{\partial}{\partial z^{1}} \wedge \cdots \wedge \frac{\partial}{\partial z^{n-1}} .
$$

Let us denote $h(y):=-f\left(\frac{1}{y}, z^{\prime}+\lambda\left(\frac{1}{y}\right)\right) y^{2}$. The group action of $\mathbb{C}$ now is

$$
\mu \cdot y=\frac{y}{1+\mu y} .
$$

The invariance of $D_{\mathfrak{g}}$ under $G$ implies for $\mu \in \mathbb{C}$ that $\mu^{*} s=c(\mu) s$, hence

$$
h(\mu \cdot y)(1+\mu y)^{2}=c(\mu) h(y) .
$$


Since $c(\mu+\kappa) s=(\mu+\kappa)^{*} s=\mu^{*} \kappa^{*} s=c(\mu) c(\kappa) s$, we obtain $c(\mu)=\exp (\rho \mu)$. This implies

$$
h(\mu \cdot y)=\frac{\exp (\rho \mu)}{(1+\mu y)^{2}} h(y) .
$$

Now fixing $y=1$ yields

$$
h\left(\frac{1}{1+\mu}\right)=c \frac{\exp (\rho(1+\mu))}{(1+\mu)^{2}}
$$

hence

$$
h(y)=c y^{2} \exp \left(\frac{\rho}{y}\right) .
$$

Since we have the additional requirement that $s \mid D_{\mathfrak{g}}=0$ and $\frac{\partial}{\partial y} \wedge \frac{\partial}{\partial z^{1}} \wedge \cdots \wedge \frac{\partial}{\partial z^{n-1}}(p)$ has a finite vanishing order, we conclude $\rho=0$. Now we see that $h$ vanishes of order 2 in 0 . We cannot guarantee that different choices of $z^{\prime}$ lead to different limit points on $D_{\mathfrak{g}}$, i.e. maybe $\frac{\partial}{\partial y} \wedge \frac{\partial}{\partial z^{1}} \wedge \cdots \wedge \frac{\partial}{\partial z^{n-1}}(p)=0$. Hence we only conclude that the vanishing order of $s$ on the limit point $p$ is at least 2. Since we could do this construction for every point of a component containing $p$, we conclude that this component is multiple.

Corollary 4.7. If $X$ is an n-dimensional projective compact complex manifold such that $\left|-m K_{X}\right|$ is base point free for some $m>0$, further $D \in\left|-K_{X}\right|$ a reduced divisor and $g$ a Kähler metric on $X \backslash D$ with divisorial invariance group $\operatorname{Aut}(X, D, g)$, then $X=T \times P$, where $P$ denotes a projective toric variety; in this case $D=\sum T \times D_{i}$, where $D_{i}$ are the distinct toric divisors of $P$.

Proof. We know $X=P \times T$. Since $D$ is reduced, $X \backslash D=\left(\mathbb{C}^{*}\right)^{l} \times T$. Since $P$ is algebraic and has an algebraic $\left(\mathbb{C}^{*}\right)^{l}$-action, it is a toric variety and $D$ is like described (cf. $[\mathrm{Fu}]$ ).

This splitting behavior cannot be expected in general. However, $X$ is always a fibre bundle over $\operatorname{Alb}(X)$. This is generally known and easy to be seen by the universal property of $\alpha$.

4.2. Non-triviality of the $\boldsymbol{G}$-Kähler cone. Recall the definition of the $G$-Kähler cone: We call two $G$-invariant Kähler metrics $g$ and $g^{\prime}$ equivalent, if there is a function $\phi \in C^{\infty}\left(X \backslash D_{\mathfrak{g}}\right)$ such that $\omega_{g}-\omega_{g^{\prime}}=i \partial \bar{\partial} \phi$. Since there is no $\partial \bar{\partial}$-lemma in the non-compact case, this cannot be viewed as the Kähler class of $\omega_{g}$, but the philosophy is very similar.

We identify again $X \backslash D_{\mathfrak{g}}=\mathbb{C}^{n} / \Lambda$. Every $\lambda_{(1)}, \lambda_{(2)} \in \Lambda$ generate a parallelogram, whose image in $X \backslash D_{\mathfrak{g}}$ is a compact real surface $T_{\lambda_{(1)}, \lambda_{(2)}}$. If $\omega=i \partial \bar{\partial} \phi$, then Stokes' 
Theorem implies

$$
0=\int_{T_{\lambda_{(1)}, \lambda(2)}} \omega=\omega_{i j}\left(\lambda_{(1)}^{i} \overline{\lambda_{(2)}^{j}}-\lambda_{(2)}^{i} \overline{\lambda_{(1)}^{j}}\right)=2 \operatorname{Im}\left(\lambda_{(1)}^{t} \underline{\omega} \overline{\lambda(2)}\right)
$$

if $\underline{\omega}=\left(\omega_{i j}\right)_{i, j}$. Note that the $\omega_{i j}$ are constant. This property only depends on $\Lambda_{\mathbb{R}}:=\Lambda \otimes \mathbb{R}$. It is easy to see that in appropriate complex coordinates every real subspace of $\mathbb{C}^{n}$ is of the form

$$
\Lambda_{\mathbb{R}}=\left\{z^{1}=\cdots=z^{l^{\prime}}=\operatorname{Im} z^{l^{\prime}+1}=\cdots=\operatorname{Im} z^{k^{\prime}}=0\right\} .
$$

In other words, $\Lambda_{\mathbb{R}}$ is generated by the real standard basis of $\mathbb{C}^{k}=\mathbb{R}^{2 k}$ and the standard basis of $\mathbb{R}^{l}=\operatorname{Re}\left(\mathbb{C}^{l}\right)$ (for $k=n-k^{\prime}, l=k^{\prime}-l^{\prime}$ ). By this choice the above equations mean that in the standard basis of $\mathbb{C}^{n}=\mathbb{C}^{k} \oplus \mathbb{C}^{l} \oplus \mathbb{C}^{n-k-l}$

$$
\underline{\omega}=\left(\begin{array}{ccc}
0 & 0 & * \\
0 & \text { real } & * \\
* & * & *
\end{array}\right),
$$

where every entry stands for the block corresponding to the factors of $\mathbb{C}^{n}=\mathbb{C}^{k} \oplus$ $\mathbb{C}^{l} \oplus \mathbb{C}^{n-k-l}$ and $*$ means that there is no claim about this entry.

Now let us reverse the direction. For the sake of simplicity, let us denote $\boldsymbol{k}:=$ $\{1, \ldots, k\}, \boldsymbol{l}:=\{k+1, \ldots, k+l\}, \boldsymbol{m}:=\{k+l+1, \ldots, n\}$. If $\omega$ is of the above form, we define $\phi$ by

$$
\begin{aligned}
\phi(z):= & 2 \sum_{i \in \boldsymbol{l}} \omega_{i i} \operatorname{Im}\left(z^{i}\right)^{2}+4 \sum_{i<j \in \boldsymbol{l}} \omega_{i j} \operatorname{Im}\left(z^{i}\right) \operatorname{Im}\left(z^{j}\right) \\
& +4 \sum_{i \in \boldsymbol{l}, j \in \boldsymbol{m}}\left(\omega_{i j} \operatorname{Im}\left(z^{i}\right) \overline{z^{j}}-\omega_{j i} \operatorname{Im}\left(z^{i}\right) z^{j}\right) \\
& +4 \sum_{i \in \boldsymbol{m}} \omega_{i i}\left|z^{i}\right|^{2}+2 \sum_{i<j \in \boldsymbol{m}} \omega_{i j} z^{i} \overline{z^{j}}
\end{aligned}
$$

then

$$
\omega-i \partial \bar{\partial} \phi=\left(\begin{array}{ccc}
0 & 0 & * \\
0 & 0 & 0 \\
* & 0 & 0
\end{array}\right) .
$$

If we now assume further that $G$ is a semi-torus (e.g. $D_{\mathfrak{g}}$ is reduced), then the factor $\mathbb{C}^{n-k-l}$ does not occur and hence

$$
\omega=i \partial \bar{\partial} \phi .
$$

This leads to the following result. 
Theorem 4.8. Let $X$ be an $n$-dimensional compact complex manifold and $G=\mathbb{C}^{n} / \Lambda$ a divisorial semi-torus. We write $\Lambda_{\mathbb{R}}=\mathbb{C}^{k} \oplus \operatorname{Re}\left(\mathbb{C}^{l}\right)$ (with $\left.k+l=n\right)$. Denote $i: M(l, \mathbb{C}) \rightarrow M(n, \mathbb{C})$ the embedding which fills up an $l \times l$-matrix with zeroes. Then

$$
K_{G}\left(X, D_{\mathfrak{g}}\right) \subset M(n, \mathbb{C}) / i(M(l, \mathbb{R}))
$$

is the cone generated by positive definite hermitian matrices. In particular, $\operatorname{dim} K_{G}\left(X, D_{\mathfrak{g}}\right)=n^{2}-\frac{1}{2} l(l+1)$.

This contrasts to the case of a smooth divisor $D$. In the appendix we will show that the Kähler cone is trivial, if $X$ has simple topology and $D$ is smooth.

Remark 4.9. The preceding calculations also show that $K_{G}\left(X, D_{\mathfrak{g}}\right)=0$ for an abelian divisorial $G$, if and only if $G=\mathbb{C}^{*} \times \mathbb{C}^{n-1}$ or $G=\mathbb{C}^{n}$. This is the case, for instance, if $X=\mathbb{P}^{n}$ and $D$ consists of two hyperplanes, one of them with multiplicity $n$ or $D$ is a hyperplane of multiplicity $n+1$, respectively. So the $G$-Kähler cone can be trivial for reducible non-reduced $D$, but only for very special cases. Note that the appearance of at most one factor $\mathbb{C}^{*}$ in $G$ is reflected in the condition $b_{2}(\tilde{X})=0$ in Theorem A.1.

\section{Example: $X=\mathbb{P}^{2}$}

If $X=\mathbb{P}^{2}$, then the tangent bundle may be described by the vector fields homogeneous of degree 1 divided by the vector fields parallel to the orbits of the group action $z \mapsto c z$, i.e. $\mathcal{O}_{X} \cdot\left(z^{0} \frac{\partial}{\partial z^{0}}+z^{1} \frac{\partial}{\partial z^{1}}+z^{2} \frac{\partial}{\partial z^{2}}\right)$. Hence the global vector fields are

$$
H^{0}\left(T_{X}\right) \cong\left(l^{0} \frac{\partial}{\partial z^{0}}+l^{1} \frac{\partial}{\partial z^{1}}+l^{2} \frac{\partial}{\partial z^{2}}\right) / \mathbb{C} \cdot\left(z^{0} \frac{\partial}{\partial z^{0}}+z^{1} \frac{\partial}{\partial z^{1}}+z^{2} \frac{\partial}{\partial z^{2}}\right),
$$

where $l^{i}$ are homogeneous linear forms. Now let $V:=\mathbb{C} v^{(1)} \oplus \mathbb{C} v^{(2)} \subset H^{0}\left(T_{X}\right)$ with $v^{(j)}=\left[\sum_{i} l^{j i} \frac{\partial}{\partial z^{i}}\right]$. In order to compute

$$
D:=\left\{z \mid v^{(1)} \wedge v^{(2)}=0\right\},
$$

we first localize to $U_{0}$ and then homogenize the result again. This procedure yields

$$
D=\left\{\operatorname{det}\left(\begin{array}{ccc}
z^{0} & z^{1} & z^{2} \\
l^{10} & l^{11} & l^{12} \\
l^{20} & l^{21} & l^{22}
\end{array}\right)=0\right\}
$$

Now let us assume that $\left[v^{(1)}, v^{(2)}\right]=0$ and $v^{(1)} \wedge v^{(2)} \not \equiv 0$. Denote $G:=$ $\exp (V)=\operatorname{Aut}^{0}(X, D)$. By assumption $G$ is divisorial and abelian, hence the 
metric $g_{\mathscr{B}}$ is Kähler and $G=\operatorname{Aut}\left(X, D, g_{\mathcal{B}}\right)$. If $D$ is reduced, Theorem 4.7 tells us that $D$ is the union of three lines in general position. If $D$ is not reduced we obtain a triple line or the sum of a line and a double line. So the only position of three lines not occurring in this list is that they are intersecting in one common point. We will now see how this corresponds to a $G$ which acts not almost transitively. After a change of coordinates we may assume that the three lines intersect in $[1: 0: 0]$. Let $v^{(1)}:=z^{1} \frac{\partial}{\partial z^{0}}, v^{(2)}:=z^{2} \frac{\partial}{\partial z^{0}}$. Of course, $\left[v^{(1)}, v^{(2)}\right]=0$ and $v^{(1)} \wedge v^{(2)} \equiv 0$, hence $G$ is abelian (indeed, $G \cong \mathbb{C}^{2}$ ) and acts not almost transitively. $G$ is given by the matrices

$$
\left(\begin{array}{lll}
1 & a & b \\
0 & 1 & 0 \\
0 & 0 & 1
\end{array}\right), \quad a, b \in \mathbb{C} .
$$

It is not hard to see that $G$ leaves $\{f=0\}$ invariant for a homogeneous $f \in$ $\mathbb{C}\left[z^{0}, z^{1}, z^{2}\right]$ if and only if $f=f\left(z^{1}, z^{2}\right)$. This factors into linear terms. Hence $G$ leaves all lines through $[1: 0: 0]$ invariant. So the not almost transitively case corresponds to the existence of a family of invariant divisors, which are not necessarily anticanonical. If $D$ consists of three lines intersecting in $[1: 0: 0]$, the full automorphism group $\operatorname{Aut}(X, D)$ consists of matrices

$$
\left(\begin{array}{lll}
c & a & b \\
0 & 1 & 0 \\
0 & 0 & 1
\end{array}\right), \quad a, b, c \in \mathbb{C},
$$

hence is not abelian and acts not almost transitively in accordance with Lemma 3.2; also $\operatorname{dim} \operatorname{Aut}(X, D)>\operatorname{dim} X$.

Back to the almost transitive $G$ and reduced $D$. Let us choose coordinates such that $D=\left\{z^{0} z^{1} z^{2}=0\right\}$. Of course, $D$ is invariant under the group $G$ given by $\left[z^{0}: z^{1}: z^{2}\right] \mapsto\left[a_{0} z^{0}: a_{1} z^{1}: a_{2} z^{2}\right]$, with $a=\left[a_{0}: a_{1}: a_{2}\right] \in \mathbb{P}^{2} \backslash\left\{a_{0} a_{1} a_{2}=0\right\} \cong$ $\mathbb{C}^{*} \times \mathbb{C}^{*}$. The group $G$ is abelian and divisorial. Theorem 3.4 and Lemma 3.1 tell us that $G=\operatorname{Aut}^{0}(X, D)$. Lemma 4.2 states that every $G$-invariant Kähler metric on $X \backslash D$ is given by a basis of $\mathfrak{g}$. Carrying out the calculations in the chart $U_{0}=\left\{z_{0} \neq 0\right\}$ yields that all $G$-invariant Kähler metrics on $X \backslash D \cong \mathbb{C}^{*} \times \mathbb{C}^{*}$ are of the form

$$
g=g_{C}=\sum_{i, j=1,2} c_{i j} \frac{d x^{i}}{x^{i}} \otimes \frac{d \overline{x^{j}}}{\overline{x^{j}}}
$$

with $c_{i j}=\overline{c_{j i}}$ and $C=\left(c_{i j}\right)>0$.

According to Theorem 4.8

$$
K_{G}(X, D) \subset M(2, \mathbb{C}) / M(2, \mathbb{R})
$$


is given by the classes of positive, hermitian matrices. Hence $K_{G}(X, D)$ is onedimensional. Moreover

$$
C(r):=\left(\begin{array}{cc}
\cosh (r) & i \sinh (r) \\
-i \sinh (r) & \cosh (r)
\end{array}\right)
$$

for $r \in \mathbb{R}$ represent every class in $K_{G}(X, D)$ uniquely.

If $X=\mathbb{P}^{3}$, then for the corresponding construction $\operatorname{dim} K_{G}\left(\mathbb{P}^{3}, D\right)=3$. In the appendix it is proved that $K\left(\mathbb{P}^{3}, D\right)=0$, if $D$ is chosen to be smooth.

\section{A. Triviality of the Kähler cone when $D$ is smooth}

Theorem A.1. Let $\tilde{X}$ be a Stein manifold with $b_{2}(\tilde{X})=0$. Then any two Kähler metrics on $\tilde{X}$ are $(\partial \bar{\partial})$-equivalent.

Proof. The injective resolution of $\mathbb{C}$

$$
0 \longrightarrow \mathbb{C} \longrightarrow \mathcal{O}_{\tilde{X}} \stackrel{\partial}{\longrightarrow} \Omega_{\tilde{X}}^{1} \stackrel{\partial}{\longrightarrow} \Omega_{\tilde{X}}^{2} \stackrel{\partial}{\longrightarrow} \cdots
$$

yields short exact sequences

$$
0 \longrightarrow \mathbb{C} \longrightarrow \mathcal{O}_{\tilde{X}} \stackrel{\partial}{\longrightarrow} \mathcal{H}_{\tilde{X}}^{1} \longrightarrow 0
$$

and

$$
0 \longrightarrow \mathcal{H}_{\tilde{X}}^{1} \longrightarrow \Omega_{\tilde{X}}^{1} \longrightarrow \mathcal{H}_{\tilde{X}}^{2} \longrightarrow 0
$$

In cohomology we obtain

$$
H^{1}\left(\mathcal{O}_{\tilde{X}}\right) \longrightarrow H^{1}\left(\mathcal{H}_{\tilde{X}}^{1}\right) \longrightarrow H^{2}(\tilde{X}, \mathbb{C}) \longrightarrow H^{2}\left(\mathcal{O}_{\tilde{X}}\right)
$$

Since $\tilde{X}$ is Stein we obtain $H^{1}\left(\mathcal{O}_{\tilde{X}}\right)=H^{2}\left(\mathcal{O}_{\tilde{X}}\right)=0$, hence

$$
H^{1}\left(\mathscr{H}_{\tilde{X}}^{1}\right)=H^{2}(\tilde{X}, \mathbb{C})=0 .
$$

The second short exact sequence yields

$$
H^{0}\left(\Omega_{\tilde{X}}^{1}\right) \longrightarrow H^{0}\left(\mathscr{H}_{\tilde{X}}^{2}\right) \longrightarrow H^{1}\left(\mathcal{H}_{\tilde{X}}^{1}\right)=0
$$

hence for every holomorphic 2-form $\eta$ on $\tilde{X}$ with $\partial \eta=0$ there is a holomorphic 1-form $\varphi$ such that $\eta=\partial \varphi$.

Now let $\omega$ be a Kähler form. Since $\Omega_{\tilde{X}}^{1}$ is coherent, Cartan's Theorem B implies $H^{1}\left(\Omega_{\tilde{X}}^{1}\right)=0$. Using the Dolbeault interpretation we obtain $\eta \in \mathcal{E}^{1,0}(\tilde{X})$ such that 
$\omega=\bar{\partial} \eta$. Now look at $\psi:=\partial \eta$. Since $0=\partial \omega=-\bar{\partial} \psi$, we conclude that $\psi \in H^{0}\left(\Omega_{\tilde{X}}^{2}\right)$. Of course, it satisfies $\partial \psi=0$. Hence there is a $\varphi \in H^{0}\left(\Omega_{\tilde{X}}^{1}\right)$ such that $\psi=\partial \varphi$. This implies $\partial(\eta-\varphi)=0$, so $\bar{\eta}-\bar{\varphi}$ induces a class in $H^{0,1}(\tilde{X})=$ $H^{1}\left(\mathcal{O}_{\tilde{X}}\right)=0$. Hence we obtain a function $G: \tilde{X} \rightarrow \mathbb{C}$ such that $\bar{\partial} G=\bar{\eta}-\bar{\varphi}$, equivalently

$$
\partial \bar{G}=\eta-\varphi
$$

For the Kähler form this means

$$
\omega=\bar{\partial} \eta=\bar{\partial}(\partial \bar{G}+\varphi)=\partial \bar{\partial}(-\bar{G}) .
$$

Since $\omega=\bar{\omega}$ we find

$$
\omega=i \partial \bar{\partial} \operatorname{Im}(G) .
$$

In the remainder of the appendix we want to show that complements of smooth ample divisors are examples for Theorem A.1, if the topology of $X$ is simple enough.

Corollary A.2. Let $X$ be a projective complex manifold with $\operatorname{dim} X \geq 3, b_{1}(X)=$ $b_{3}(X)=0, b_{2}(X)=1$ and $D \subset X$ a smooth ample divisor. Then $K(X, D)=0$.

The proof consists of the verification of $b_{2}(X \backslash D)=0$. We have to prove $b_{1}(X \backslash D)=0$ first in order to be able to prove $b_{2}(X \backslash D)=0$, however. In the following we abbreviate $\tilde{X}:=X \backslash D$. Of course, since $\tilde{X}$ is quasi-projective, $\tilde{X}$ is Kähler. So $K(X, D) \neq \emptyset$.

Lemma A.3. If $X$ is a projective complex manifold with $\operatorname{dim} X \geq 3, b_{1}(X)=0$ and $D \subset X$ a smooth ample divisor, then $b_{1}(\tilde{X})=0$.

Proof. First we construct a $S^{1}$-principal tubular neighbourhood of $D$. This is done by choosing a Kähler metric $\omega$ on $X$ and defining the fibre of $U(D) \rightarrow D$ over $z \in D$ as the image of

$$
\exp _{z}: B_{\varepsilon} \longrightarrow X
$$

where $B_{\varepsilon}:=\left\{v \in\left(T_{z} D\right)^{\perp_{\omega}} \mid \omega(v, v) \leq \varepsilon^{2}\right\}$. For small enough $\varepsilon$ this yields a $C^{\infty}$ disk bundle embedded in $X$ with a naturally given $S^{1}$ action. $E:=\partial U(D)$ is now a principal $S^{1}$-bundle $\pi: E \rightarrow D$.

Since $\tilde{X} \cap U(D)=U(D) \backslash D$ has $E \rightarrow D$ as a deformation retract and $U(D)$ is contractible to $D$, Mayer-Vietoris yields

$$
H^{1}(X, \mathbb{C}) \longrightarrow H^{1}(\tilde{X}, \mathbb{C}) \oplus H^{1}(D, \mathbb{C}) \longrightarrow H^{1}(E, \mathbb{C}) .
$$

Since by assumption $H^{1}(X, \mathbb{C})=0$, it is enough to show $H^{1}(E, \mathbb{C})=0$ in order to prove the claim of the lemma. 
So we consider a closed one-form $\eta \in A^{1}(E)$. We refer to [GHV, Prop. X, p. 304] for the existence of a fibre-wise integration

$$
\int_{E_{z}}: A^{p}(E) \longrightarrow A^{p-1}(D)
$$

commuting with the differential $d$. In particular,

$$
c:=\int_{E_{z}} \eta
$$

is a constant.

Now we go down to local coordinates: Let $D=\bigcup U_{(j)}$ be a trivializing covering with coordinates $\left(z_{(j)}^{k}\right)$. We may assume that $U_{(j)}$ are simply connected and $U_{(i j)}:=$ $U_{(i)} \cap U_{(j)}$ are connected. The arc coordinate on $S^{1} \times U_{(j)}$ is denoted by $\phi_{(j)}$. The one-form $\eta$ is now represented by

$$
\eta_{0(j)} d \phi_{(j)}+\sum_{k} \eta_{k(j)} d z_{(j)}^{k} .
$$

The fibre-wise Fourier series of $\eta$ looks like

$$
\sum_{r=-\infty}^{\infty} \eta_{0 r(j)} \exp \left(i r \phi_{(j)}\right) d \phi_{(j)}+\sum_{r, k} \eta_{k r(j)} \exp \left(\operatorname{ir} \phi_{(j)}\right) d z_{(j)}^{k}
$$

with $\eta_{l r(j)}: U_{(j)} \rightarrow \mathbb{C}$. We want to collect terms of the same degree $r$,

$$
\eta_{r}:=\eta_{0 r(j)} \exp \left(i r \phi_{(j)}\right) d \phi_{(j)}+\sum_{k} \eta_{k r(j)} \exp \left(\operatorname{ir} \phi_{(j)}\right) d z_{(j)}^{k} .
$$

In order to prove independence of $j$ we have to look closer at the transition maps.

The transition maps $S^{1} \times U_{(i j)} \rightarrow S^{1} \times U_{(i j)}$ are given by

$$
\left(\exp \left(i \phi_{(i)}\right), z_{(i)}^{k}\right) \mapsto\left(\beta_{(i j)}(z) \exp \left(i \phi_{(i)}\right), z_{(j)}^{k}\right)
$$

with $\beta_{(i j)}: U_{(i j)} \rightarrow S^{1}$ due to the construction of $E$. This transition law implies

$$
\eta_{0 r(i)}=\beta_{(i j)}^{r} \eta_{0 r(j)}
$$

hence $h_{r(j)}:=\eta_{0 r(j)} \exp \left(\operatorname{ir} \phi_{(j)}\right)$ defines a global function $h_{r}: E \rightarrow \mathbb{C}$. Moreover,

$$
d h_{r}=i r \eta_{r},
$$

hence $\eta_{r}$ is well defined for any $r \geq 0$. At the same time this shows that $\eta-\eta_{0}$ is exact, hence only $\eta_{0}$ remains to be considered. First note that

$$
c=\int_{E_{z}} \eta=\int_{E_{z}} \eta_{0}=2 \pi \eta_{00}(j)
$$


independently of $j$. For the other terms we know $0=d\left(\eta_{0}-\frac{c}{2 \pi} d \phi_{(j)}\right)$, hence there are $f_{(j)}: U_{(j)} \rightarrow \mathbb{R}$ such that

$$
\frac{c}{2 \pi} d \phi_{(i)}+d f_{(i)}=\frac{c}{2 \pi} d \phi_{(j)}+d f_{(j)} .
$$

The universal coefficient lemma tells us that $H^{1}\left(D, S^{1}\right)=H_{1}(D, \mathbb{Z})$ (not canonically), since by the Lefschetz theorem there is no free part of $H_{1}(D, \mathbb{Z})$. This is a finite group, so there exists some $m$ such that $m g=0$ for all $g \in H^{1}\left(D, S^{1}\right)$.

If $c \neq 0$, we interpret $f_{(j)}: U_{(j)} \rightarrow \mathbb{R} / c \mathbb{Z} \cong S^{1}$ as a circle valued function and infer that there are $c_{(j)} \in S^{1}$ such that

$$
m\left(\frac{c}{2 \pi} \phi_{(j)}+f_{(j)}(z)\right)+c_{(j)}
$$

represents a global function $\Phi: E \rightarrow S^{1}$. The level sets of $\Phi$ induce nowhere vanishing $C^{\infty}$ sections of $N_{D \mid X}^{\otimes m}$, so $N_{D \mid X}^{\otimes m} \cong \mathcal{O}_{D}$ differentiably. This clearly contradicts the assumption that $D$ is ample.

So $c=0$ and hence $\eta_{0}=\pi^{*} \zeta$ for some $\zeta \in A^{1}(D)$. Since we know $H^{1}(D, \mathbb{R})=0$, we infer that $\eta_{0}$ is exact and hence $\eta$ is exact.

Lemma A.4. Under the assumptions of Corollary A.2 holds $b_{2}(\tilde{X})=0$.

Proof. We choose the same tubular neighbourhood like in the proof of Lemma A.3 and use Mayer-Vietoris for $X=\tilde{X} \cup U(D)$. Recall that we already proved $H^{1}(E, \mathbb{C})=0$. So we obtain

$$
0 \longrightarrow H^{2}(X, \mathbb{C}) \longrightarrow H^{2}(\tilde{X}, \mathbb{C}) \oplus H^{2}(D, \mathbb{C}) \longrightarrow H^{2}(E, \mathbb{C}) \longrightarrow H^{3}(X, \mathbb{C}) .
$$

In order to compute the cohomology of $E$ we use the Leray spectral sequence. For a circle bundle, according to [S, 9.5, Thm2] this simplifies in our case to

$$
0 \longrightarrow H^{0}(D, \mathbb{C}) \longrightarrow H^{2}(D, \mathbb{C}) \longrightarrow H^{2}(E, \mathbb{C}) \longrightarrow H^{1}(D, \mathbb{C}) .
$$

Like before we use the Lefschetz theorem to conclude $h^{1}(D, \mathbb{C})=0$. Hence $b_{2}(E)=$ $b_{2}(D)-1$. If we now use the assumptions $b_{3}(X)=0$ and $b_{2}(X)=1$, then the sequence implies $b_{2}(\tilde{X})=0$.

Acknowledgments. The authors are grateful to Hans-Christoph Grunau and Georg Schumacher for proposing and supporting the project. 
Vol. 81 (2006)

\section{References}

[BK88] S. Bando, R. Kobayashi, Ricci-flat Kähler metrics on affine algebraic manifolds. In Geometry and analysis on manifolds (Katata/Kyoto, 1987), 20-31, Lecture Notes in Math. 1339, Springer-Verlag, Berlin 1988. Zbl 0659.53048 MR 0961470

[BK90] S. Bando, R. Kobayashi, Ricci-flat Kähler metrics on affine algebraic manifolds. II. Math. Ann. 287 (1990), 175-180. Zbl 0701.53083 MR 1048287

[B156] A. Blanchard, Sur les variétés analytiques complexes. Ann. Sci. Ecole Norm. Sup. (3) 73 (1956), 157-202. Zbl 0073.37503 MR 0087184

[Fu] W. Fulton, Introduction to Toric Varieties. Ann. of Math. Stud. 131, Princeton University Press, Princeton, NJ, 1993. Zbl 0813.14039 MR 1234037

[GHV] W. Greub, S. Halperin, R. Vanstone, Connections, curvature, and cohomology. Vol. I, Pure and Appl. Math. 47, Academic Press, New York, London 1972. Zbl 0322.58001 MR 0336650

[La] S. Lang, Differential and Riemannian manifolds. Grad. Texts in Math. 160, SpringerVerlag, New York, Berlin, Heidelberg 1995. Zbl 0824.58003 MR 1335233

[On1] A. L. Onishchik, R. V. Gamkrelidze (eds.), Lie Groups and Lie Algebras I. Encyclopaedia Math. Sci. 20, Springer-Verlag, Berlin, New York, London 1993. Zbl 0777.00023 MR 1306737

[Ma92] B. Maßmann, Equivariant Kähler compactifications of homogeneous manifolds. $J$. Geom. Anal. 2 (1992) , 555-574. Zbl 0768.32019 MR 1189044

[MM60] Y. Matsushima, A. Morimoto, Sur certains espaces fibrés holomorphes sur une variété de Stein. Bull. Soc. Math. France 88 (1960), 137-155 (in French). Zbl 0094.28104 MR 0123739

[Mo66] A. Morimoto, On the classification of noncompact complex abelian Lie groups. Trans. Amer. Math. Soc. 123 (1966), 200-228. Zbl 0144.07903 MR 0207893

[S] E. Spanier, Algebraic Topology. McGraw-Hill, New York, Toronto, Ont.-London 1966. Zbl 0145.43303 MR 0210112

[TY90] G. Tian, S.-T. Yau, Complete Kähler manifolds with zero Ricci curvature. I. J. Amer. Math. Soc. 3 (1990), 579-609. Zbl 0719.53041 MR 1040196

[TY91] G. Tian, S.-T. Yau, Complete Kähler manifolds with zero Ricci curvature. II. Invent Math. 106 (1991), 27-60. Zbl 0766.53053 MR 1123371

[Wi04] J. Winkelmann, On manifolds with trivial logarithmic tangent bundle. Osaka J. Math. 41 (2004), 473-484. Zbl 1058.32011 MR 2069097

Received August 21, 2003; revised February 8, 2004

Bert Koehler, Philipps-Universität Marburg, Department of Mathematics, 35032 Marburg, Germany

E-mail: koehlerb@mathematik.uni-marburg.de

Marco Kühnel, Otto-von-Guericke-Universität Magdeburg, FMA/IAN, Postfach 4120, 39016 Magdeburg, Germany

E-mail: Marco.Kuehnel@ Mathematik.Uni-Magdeburg.de 